\section{FutureJournal}

FUTURE STUDIES RESEARCH JOURNAL: TRENDS AND STRATEGIES

\title{
Um Modelo para Auxiliar na Tomada de Decisão no Domínio de Gestão de Ideias
}

\author{
Marina Carradore Sérgio \\ Mestranda em Engenharia e Gestão de Conhecimento pela Universidade Federal de Santa Catarina \\ (UFSC), Brasil \\ marinacarradore@egc.ufsc.br
}

\begin{abstract}
Alexandre Leopoldo Gonçalves
Doutor em Engenharia de Produção pela Universidade Federal de Santa Catarina (UFSC), Brasil a.I.goncalves@ufsc.br
\end{abstract}

\section{João Artur de Souza}

Doutor em Engenharia de Produção pela Universidade Federal de Santa Catarina (UFSC), Brasil joao.artur@ufsc.br

\section{RESUMO}

Diante da competitividade global, inovar tornou-se um desafio para as organizações. A gestão de ideias é parte integrante do processo de inovação e apresenta-se como um fator essencial para obtenção de sucesso do mesmo. Devido ao volume e aos picos repentinos de submissões de ideias, a adequada análise e a destinação de recursos para investimentos são questões importantes a serem tratadas. O objetivo deste trabalho é apresentar um modelo para a gestão de ideias baseado em ontologia e na análise de agrupamento visando maximizar os recursos para investimentos em ideias. De modo a demonstrar a viabilidade do modelo elaborou-se um conjunto de dados de cinquenta e cinco ideias coletadas a partir do site da empresa Starbucks $®$. Estas ideias foram então armazenadas em uma ontologia de domínio servindo de subsídio para a análise de agrupamento e a construção de uma base de conhecimento. Como resultado, foram identificados grupos de ideias semelhantes que ao serem analisados promovem um maior potencial de observação e, podem indicar padrões e tendências que auxiliem na tomada de decisão.

Palavras-chave: Gestão de Inovação. Gestão de Ideias. Ontologia. Análise de Agrupamentos. 


\section{FutureJournal}

FUTURE STUDIES RESEARCH JOURNAL: TRENDS AND STRATEGIES

\section{A Model to Support Decision Making in the Idea Management Domain}

\section{ABSTRACT}

Taking into account the global competitiveness, innovation has become a challenge for organizations. Idea management is an integral part of the innovation process and it is presented as an essential factor for achieving success. Due to the volume and sudden peaks in submissions of ideas, the appropriate analysis and the allocation of resources for investment are important issues to be addressed. The objective of this paper is to present a model for the management of ideas based on ontology and cluster analysis in order to maximize resources for investment in ideas. Aiming to demonstrate the viability of the model we elaborated a dataset composed of fifty-five ideas collected from the Starbucks $\AA$ site. These ideas were then stored in a domain ontology used as subsidies for the cluster analysis and the construction of a knowledge base. As a result, we identified similar groups of ideas that promote a greater potential for observation and may indicate patterns and trends to assist in decision-making.

Key-words: Innovation Management. Idea Management. Ontology. Cluster Analysis. 


\section{INTRODUÇÃO}

A competitividade presente no cenário de mercado global faz com que as organizações busquem investir em produtos e/ou serviços inovadores. Ao desenvolver o processo de inovação, produtos são criados, objetivando atingir novos mercados, em busca da manutenção da vantagem competitiva (Reid \& Brentani, 2004). Porém inovar não é um processo trivial.

Segundo Trott (2012) inovar significa despender investimento no processo de geração de ideias, no desenvolvimento de tecnologias e processos ou ainda na fabricação e marketing de um produto. Por meio de novas ideias é possível criar novos produtos e desenvolver o processo de inovação (Witell, Löfgren \& Gustafsson, 2011). Segundo Vygotsky (2007) o principal insumo presente na sociedade é o conhecimento e a capacidade de produzir ideias inovadoras.

Barbieri, Álvares e Cajazeira (2009) afirmam que as ideias são a base do processo de inovação. Para Brem e Voigt (2007) ideias compõem a matéria-prima do processo. Endesley (2010) complementa ainda que "o coração da inovação é a geração e o teste de novas ideias". Entretanto organizações que desenvolvem inovações como fonte de estratégia competitiva devem estar preparadas para a criação e a gestão de ideias (Barbieri, Álvares \& Cajazeira, 2009).

A gestão de ideias é parte integrante do processo de inovação e pode representar o ponto inicial deste processo, auxiliando na identificação de oportunidades viáveis e plausíveis de serem implementadas por determinada organização (Björk, Boccardelli \& Magnusson, 2010; Vandenbosch, Saatcioglu \& Fay, 2006). Segundo Sint et al. (2010) e Xie e Zhang (2010) através da gestão de ideias é possível aumentar a produtividade das empresas, beneficiar a criação de novos produtos e melhorar os processos realizados na organização, minimizando os custos e maximizando a manutenção da vantagem competitiva.

O processo de gerir de ideias apresenta-se como um setor promissor para a indústria de desenvolvimento de software para coletar e organizar sugestões que podem catalisar inovações em produtos e serviços (Westerski \& Iglesias, 2011). Os sistemas de gestão de ideias apresentam-se como uma 
alternativa para gerenciar este processo. O principal objetivo do mesmo é prover ferramentas que possibilitem avaliar as ideias coletadas e eleger para implementação somente as melhores (Westerski, Iglesias \& Rico, 2010). Para Conry-Murray (2010), o interesse crescente em sistemas de gerenciamento de ideia tem motivado comunidades a desenvolverem plataformas com a finalidade de integrar o processo de inovação ao processo de software.

Todavia trabalhar com ideias inovadoras promovem desafios. Entre estes citam-se a seleção das ideias mais relevantes e passíveis de implementação e investimentos de recursos financeiros (Sint et al., 2010). Outro ponto central está em como aprimorar o processo de gestão de ideias e torná-lo mais eficiente e rápido devido a períodos com grandes sobrecargas de informação para o sistema de software (Sint et al., 2010; Westerski, Iglesias \& Rico, 2010; Westerski \& Iglesias, 2011).

O objetivo deste trabalho é o desenvolvimento de um modelo para auxiliar no processo de tomada de decisão e na seleção de ideias para serem implementadas. Com a formalização desse conhecimento objetiva-se promover um impacto positivo auxiliando no processo de Gestão de Ideias.

Segundo os autores Fernandes et al. (2011), poucas são as ferramentas computacionais existentes para apoiar o processo de inovação, sendo que as tecnologias presentes na Web Semântica, como as ontologias, representam um grande potencial para área.

Para elucidar o artigo proposto, foi realizada uma pesquisa exploratória, buscando fundamentação em uma revisão de literatura para enfatizar os métodos utilizados e definir as bases de um modelo de tratamento de ideias. A partir disso, um protótipo foi desenvolvido e aplicado em um cenário para evidenciar a eficiência da proposição. Neste sentido, é utilizada uma ontologia de domínio modelada com foco no raciocínio semântico por meio de regras e a aplicação de um processo de agrupamento baseado em uma análise textual.

As próximas seções deste artigo são organizadas da seguinte maneira: na seção 2 serão abordados os conceitos referentes ao processo de inovação, gestão de ideias, ontologia e ao processo de agrupamento. Na seção 3 será apresentado o modelo desenvolvido para o processo de gerenciamento de ideias. Os resultados da aplicação do modelo são detalhados na seção 4 com 
suas respectivas avaliações que visam apresentar alternativas para uma melhor distribuição de recursos de modo geral na Gestão de Ideias. Por fim, na seção 5 são apresentadas as considerações finais.

\section{FUNDAMENTAÇÃO TEÓRICA}

A fundamentação teórica deste artigo está centrada nos seguintes tópicos: ao processo de inovação, a gestão de ideias, a ontologia e o processo de agrupamento.

\subsection{PROCESSO DE INOVAÇÃO}

A inovação tornou-se um fator crucial para sustentar a organização no mercado (Gibson \& Skarzynski, 2008) o que representa um mecanismo complexo capaz de transformar processos em vantagens competitivas (Bautzer, 2009). Não existe um consenso sobre o significado do termo inovação (Chesbrough, 2006). A OECD (Organisation for Economic Cooperation and Development) em 2010 definiu inovação como: "a implementação de um produto (bem ou serviço) novo ou significativamente melhorado, ou um processo, ou um novo método de marketing, ou um novo método organizacional nas práticas de negócios, na organização do local de trabalho ou nas relações externas".

O autor Trott (2012) apresenta o termo inovação como o processo de geração de ideias, o desenvolvimento de tecnologias, a fabricação e marketing de um produto e o desenvolvimento de um processo. Autores como Baregheh, Rowley e Sambrook (2009) definem inovação como o processo na qual a organização, a partir das ideias, desenvolve produtos novos ou melhorados, serviços ou processos, com o intuito de crescimento, buscando competir e alcançar diferenciais para obtenção de sucesso no mercado.

Segundo Artz (2010) o termo inovação está relacionado ao desenvolvimento de produtos ou serviços comercialmente viáveis. Para os autores, é necessário que exista a distinção do processo de invenção do processo de inovação. Onde o termo invenção envolveria o desenvolvimento de novas ideias estando intimamente relacionado ao número de patentes, 
enquanto que o termo inovação consiste em originar resultados lucrativos através da efetivação das ideias.

Para Tidd, Bessant e Pavitt (2005) a gestão de inovação é "o processo de planejamento, alocação, organização e coordenação de fatores essenciais para que se alcance resultados inovadores". Na visão dos autores Gambatese e Hallowell (2011), o processo de inovação envolve diferentes componentes e atividades para originar novas ideias e transformá-las em realidade.

A inovação promove a movimentação dos resultados de investimentos em pesquisa e desenvolvimento, internamente ou externamente a organização (Badawy, 2011). Atividades que fazem uso intensivo de conhecimento como educação, inovação e P\&D apoiam o pilar do crescimento econômico (Cicyt, 2007), seja no ambiente organizacional ou ainda no âmbito global.

A inovação é intrinsicamente dependente do ambiente organizacional, necessitando do fomento ao surgimento de ideias criativas e sua possível implantação (Tidd, Bessant \& Pavitt, 2005). Contudo, é necessária a construção e manutenção do ambiente organizacional, pois só assim, efetivamente, a gestão da inovação alcançará êxito.

\subsection{GESTÃO DE IDEIAS}

Ideias são consideradas como a principal matéria-prima do processo de inovação (Brem \& Voigt, 2007). A competência das organizações em gerar inovações necessita de uma demanda contínua de ideias, objetivando desenvolver novas tecnologias, produtos e serviços (Bessant et al., 2005). Adaptar-se às mudanças reais e ao contexto de inserção é essencial no cenário de mercado atual (Govindarajan \&Trimble, 2012).

A gestão de ideias encontra-se no front end da inovação, ao qual é compreendida como o núcleo da gestão da inovação (Bothos, Apostolou \& Mentzas, 2012), e representa um processo crucial para acrescer a produtividade das organizações (Sint et al., 2010). Representa ainda valor real para as organizações, pois é considerada o ponto inicial do processo de inovação e por prover auxílio na identificação de oportunidades (Björk, Boccardelli \& Magnusson, 2010; Vandenbosch, Saatcioglu \& Fay, 2006). 
Para os autores Sint et al. (2010), Westerski e Iglesias (2011), Xie e Zhang (2010), Poveda, Westerski e Iglesias (2012) a partir da gestão de ideias é possível:

- Aumentar a produtividade das organizações;

- Melhorar os processos realizados na organização;

- Favorecer a criação de novos produtos;

- Minimizar os custos;

- Manutenção da vantagem competitiva organizacional;

- Gerar valor comercial por meio das ideias;

A oportunidade de gerar novas ideias pode emergir dos colaboradores internos ou ainda externos como fornecedores e consumidores (Boeddrich, 2004). A interação entre os colaboradores da organização é fundamental para o processo de inovação e consequentemente para o favorecimento da criação de ideias (Björk \& Magnusson, 2009; Blohm et al., 2011).

Ideias necessitam ser avaliadas de acordo com critérios como investimento, benefícios, capacidade de inovação e a relevância estratégica para a empresa (Sint et al., 2010). Em estudo recente Magnusson, Netz e Wästlund (2014) identificaram os três principais critérios para a seleção de ideias que são: a Originalidade, ao qual representa a questão de novidade e inovação; o Valor para o Usuário representando as perspectivas dos usuários sobre a implantação da ideia ou serviço e se a mesma irá gerar valor para eles; e por último o critério de Produtibilidade, representando a perspectiva do produtor em relação à facilidade com que o serviço pode ser implementado e produzido. Os autores ainda identificaram por meio da pesquisa, dois novos critérios como a adequação estratégica da ideia ou do serviço e a questão de lucratividade que a ideia poderá originar.

Porém segundo Westerski e Iglesias (2011), dentre as etapas do processo de gerir ideias, a que possui maior relevância é a avaliação dos dados, sendo ainda a mais problemática. Para os autores Westerski; Dalamagas; Iglesias (2013), os grandes desafios da área envolvem:

- O excesso de informação através do grande volume de ideias submetidas;

- Picos súbitos de submissões de ideias;

- A redundância das ideias;

- Grandes quantidades de ideias triviais; 
- Enorme esforço humano necessário durante o processo de gestão de ideias;

\subsection{ONTOLOGIA}

Conforme apresentado em Borst (1997), o termo ontologia originasse na filosofia, ao qual está relacionado ao ser ou aos tipos de existência. Na inteligência artificial o termo está associado à como um sistema de IA deve racionar para executar determinada tarefa útil.

Baseado em Gruber (1995) e Borst (1997), os autores Studer, Benjamins e Fensel (1998) apresentaram a seguinte definição: "a ontologia é a representação formal e explícita de uma conceitualização compartilhada". De acordo com Guarino (1998), a ontologia é uma forma de definir um vocabulário para descrever determinada realidade. Para Neches et al. (1991), a ontologia define os termos básicos e relações, envolvendo o vocabulário de uma determinada área bem como as regras para combinar termos e relacionamentos definindo extensões do vocabulário.

Ontologias permitem realizar a representação do conhecimento por meio de termos e definições acerca dos conceitos relacionados a um dado domínio, promovendo a representação das relações semânticas, e não somente a relação sintática dos dados. Ao desenvolver uma ontologia é necessário: descrever as categorias e os objetos bem como as relações entre os dados envolvidos (Lula \& Paliwoda-Pękosz, 2008). Possui capacidade de exploração e representação de relacionamentos semânticos e conceituais dos termos pertencentes a um determinado domínio (Silva et al., 2009). O uso de ontologias permite a habilidade em raciocinar, analisar e operar sobre o conhecimento armazenado.

Kobashi (2007) também corrobora da afirmação anterior e salienta que ontologias proveem a base necessária para o raciocínio sobre os objetos de determinado domínio. Isto ocorre devido à representação do conhecimento explicitar o comportamento dos objetos e suas relações (Kobashi, 2007).

Segundo Gómez-Pérez (1999) uma ontologia não inclui somente os termos que podem ser explicitados, mas também os que podem ser obtidos por meio de regras de inferência. A meta-informação presente na semântica pode, por exemplo, agrupar ideias em categorias, auxiliar na busca e até 
mesmo avaliação das ideias, extraindo as ideias mais fortemente discutidas ou as ideias que foram classificadas como as melhores pelo conjunto de usuários (Hüsig \& Kohn, 2011).

\subsection{ANÁLISE DE AGRUPAMENTO}

Lidar de maneira eficiente e rápida com o processo de gestão de ideias torna-se um desafio, pois ocorrem períodos com grandes sobrecargas de informação para o sistema (Sint et al., 2010; Westerski, Iglesias \& Rico, 2010; Westerski \& Iglesias, 2011). Sint et al. (2010) mencionam o desafio de encontrar as ideias mais relevantes perante o volume de ideias.

Os estudos voltados ao agrupamento de dados atendem a determinados objetivos por propiciarem informações acerca do grau de semelhança ou de diferença entre dois ou mais objetos (Vasconcelos et al., 2007). Cruz \& Carneiro (2006) afirmam que a análise de agrupamento apresenta-se como uma das melhores alternativas para realizar análises e interpretações dos dados.

A análise de agrupamento, ou seja, a descoberta e interpretação de grupos de objetos tendo propriedades e/ou comportamentos semelhantes é uma das operações mais comuns na exploração e análise de vários tipos de dados (Andrienko \& Andrienko, 2009). Segundo Jain, Murty e Flynn (1999), a análise de agrupamento consiste na organização de um conjunto de padrões (geralmente dispostos em vetores ou em espaços multidimensionais) em grupos de acordo com um grau de semelhança.

A técnica de agrupamento é útil por auxiliar na análise de grandes volumes de dados, uma vez que permite a um analista considerar grupos de objetos em vez de objetos individuais. Para Andrienko e Andrienko (2009) agrupamentos também podem ser úteis para outros fins, como na detecção de objetos incomuns, que podem exigir uma investigação especial. Os resultados podem ser utilizados para quaisquer fins, por exemplo, na tomada de decisão. A análise constitui a parte fundamental do processo, pois é por meio da interpretação dos agrupamentos por um analista humano que os mesmos adquirem significado e valor.

Existem diferentes métodos para realização do processo de agrupamento de dados. A distinção entre os métodos está relacionada ao tipo 
de resultado a ser obtido e pelas diferentes formas de definir a proximidade entre um indivíduo e um grupo já formado, ou entre dois grupos quaisquer (Vasconcelos et al., 2007).

Em um conjunto de dados, por exemplo, a mesma ideia pode ser recorrente na base, aparecendo inúmeras vezes, embora com especificidades diferentes. Em uma meta-análise pode-se identificar grupos (clusters) de ideias semelhantes, apontando para possíveis padrões e tendências. Isoladamente estas ideias podem não ser interessantes, mas o potencial aumenta quando agrupadas. Ou ainda caso a mesma ideias apareça várias vezes na base de dados, apontar para uma possível demanda por este tipo de serviço (Magnusson, Netz \& Wästlund, 2014). Os autores salientam que a formação dos agrupamentos é um fator que influência no momento da avaliação das ideias por parte dos especialistas.

A implementação de um módulo em que a informação obtida durante o processo de pesquisa, possa ser visualizada no formato de agrupamentos, poderá fazer com que especialistas interpretem de uma maneira melhor as ideias coletadas na organização (Poveda, Westerski \& Iglesias, 2012).

\section{MODELO PROPOSTO}

A concorrência entre as organizações vem promovendo uma busca constante por ideias inovadoras. Como mencionado anteriormente a gestão de ideias produz desafios no momento da avaliação dos dados, representando o ponto crítico do processo, devido ao volume de submissões ou ainda pela trivialidade das ideias. Para tanto, apresenta-se neste trabalho um modelo que auxilie na identificação de ideias inovadoras e consequentemente no processo de tomada de decisão. A Figura 1 apresenta o modelo. 


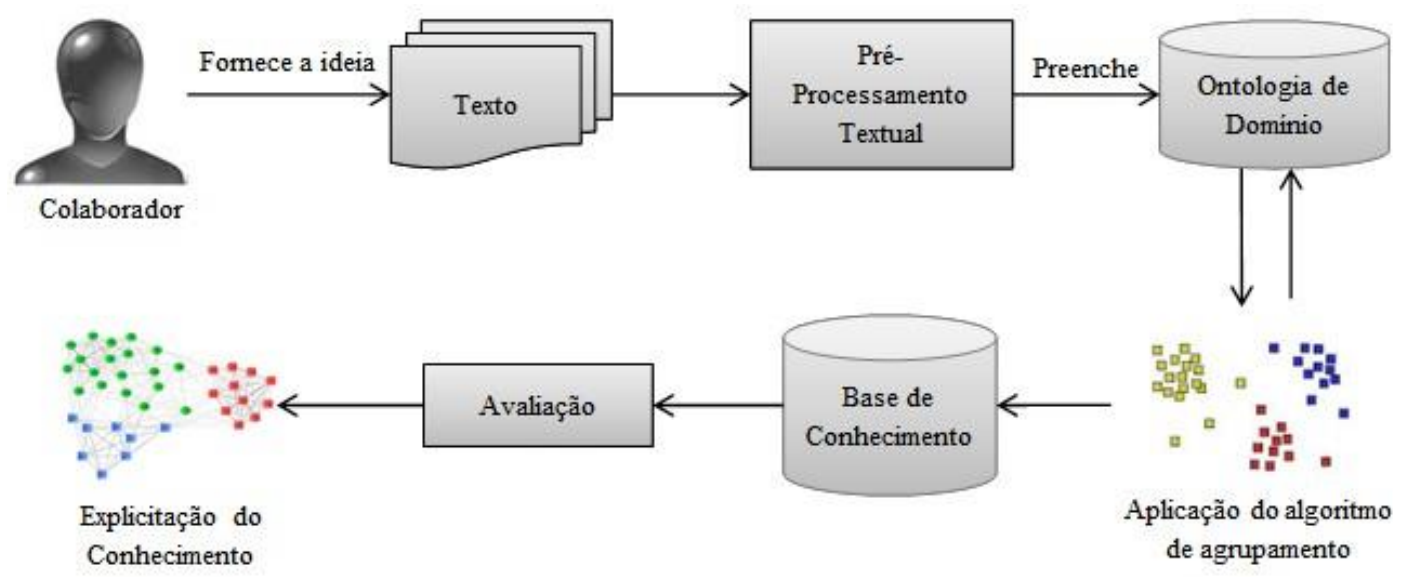

\section{Figura 1 - Modelo proposto}

Fonte: Autores

O primeiro passo apresentado no modelo compreende o preenchimento da base de dados. O colaborador da organização fornece suas ideias dispostas no formato textual, ou seja, não estruturado. Posteriormente é realizado um pré-processamento textual para adequação do texto a um formato semiestruturado, colocando-o no padrão XML. Esta fase possui como intuito transformar as ideias propostas em uma linguagem entendível aos meios computacionais.

Em uma fase subsequente é realizada a leitura dos arquivos em formato XML contendo as propostas de ideias, bem como, a indexação dos arquivos através de uma biblioteca de indexação de documentos, neste caso, Lucene Apache $\circledast$.

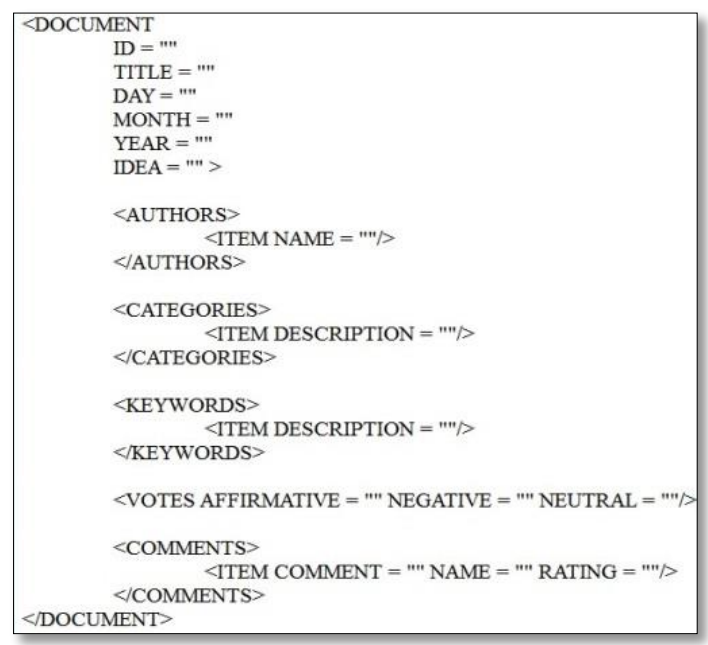

Figura 2 - Documento XML

Fonte: Autores 
Como demonstra a Figura 2 são extraídas algumas características dos documentos, entre elas, o título da ideia; o dia, o mês e o ano que a ideia foi postada; o nome do(s) autor(es); a categoria em que a ideia esta incluída; as palavras-chaves; o número de votos e o(os) comentário(s) que a ideia recebeu. As palavras-chave foram extraídas no momento da leitura dos documentos.

Em fase concomitante ao processo de indexação ocorre a população da ontologia de domínio voltada à gestão de ideias. A ontologia foi desenvolvida como forma de representação do conhecimento de domínio. Não se identificou na literatura e em pesquisas realizadas na web nenhuma ontologia com a capacidade de realização de inferência por meio de regras e com suporte ao processo de agrupamento. A Figura 3 apresenta a ontologia desenvolvida. As classes principais correspondem à ideia em si, representada na figura pela classe Idea, e a classe Cluster responsável por armazenar o resultado do processo de agrupamento. As principais propriedades de dados da classe Idea são o título da ideia, a descrição da ideia, a data de submissão da ideia e um identificador. Já a classe Cluster contém um identificador, a descrição do agrupamento, e através da propriedade de objetivo hasIdea são conectadas todas as ideias que estão presentes em determinado agrupamento. Esta conexão é realizada após a execução do processo de agrupamento de ideias. Via inferência são atribuídos ainda ao agrupamento os descritores ou palavras-chave que o definem. A regra responsável pela inferência afirma que: se uma ideia possui um descritor $\mathbf{x}$ e a mesma ideia pertence a um agrupamento (cluster) $\mathbf{y}$, então o agrupamento (cluster) y possui como descritor $\mathbf{x}$. 


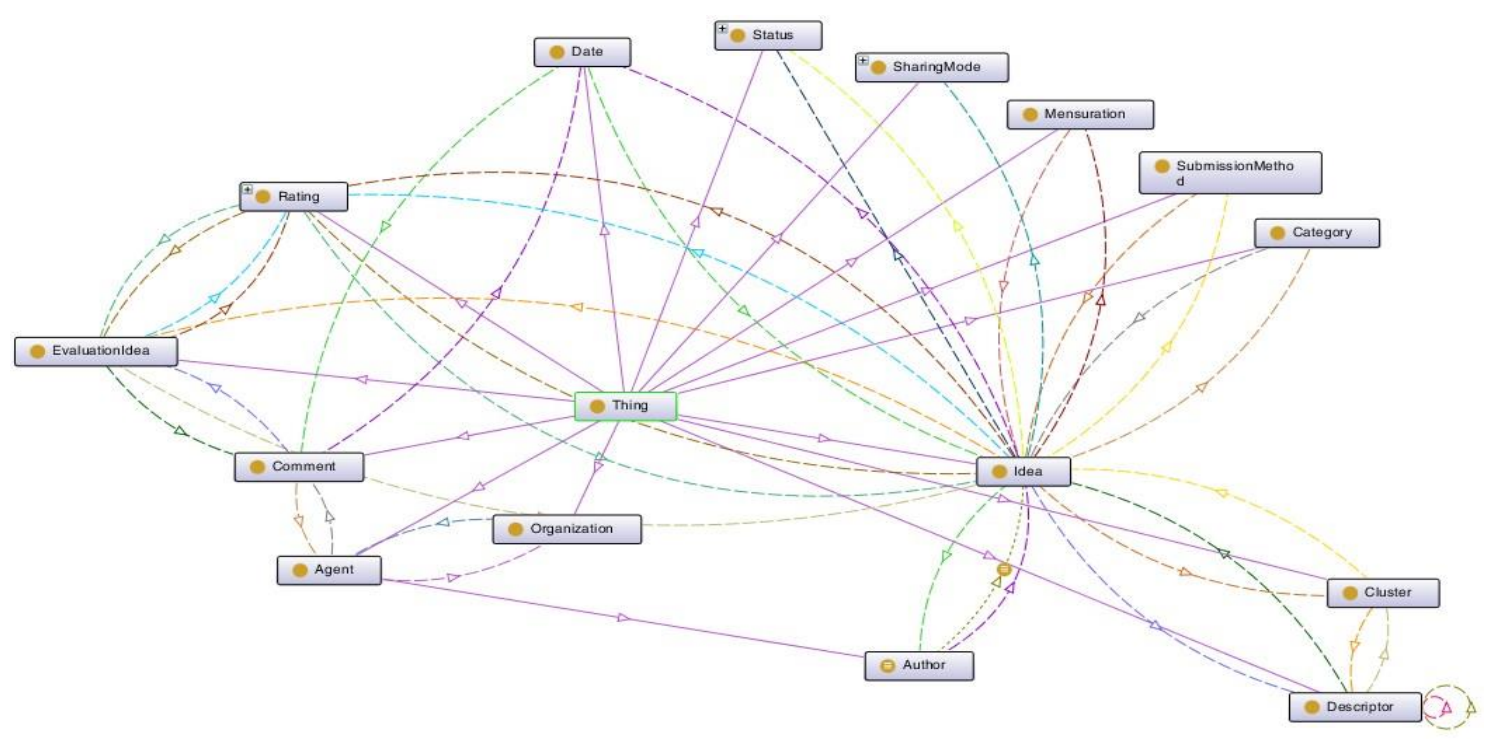

Figura 3 - Ontologia desenvolvida para gestão de ideias

Fonte: Autores

Na fase de agrupamento foi utilizado o algoritmo Lingo disponível no projeto Carrot2[1] para visualização dos agrupamentos formados em decorrência das ideias. Segundo o site do projeto, o Carrot ${ }^{2}$ é uma biblioteca com um conjunto de aplicativos para construir um mecanismo de agrupamento de resultados de pesquisa cujo objetivo é organizar os resultados da pesquisa em tópicos, de forma totalmente automática e sem conhecimentos externo, como taxonomias ou conteúdo pré-classificados.

O algoritmo Lingo é aplicado no processo de formação de agrupamentos. Segundo Osinski e Weiss (2004) a diferença do Lingo em relação a outros algoritmos está no momento da descrição do agrupamento, pois ele tenta descobrir o melhor termo que sirva de identificação de determinado agrupamento. Segundo o autor Carpineto et al (2009), o Lingo possui quatro fases sendo elas: (1) o pré-processamento de trechos; (2) a extração das frases que mais coocorrem; (3) a inserção dos rótulos nos agrupamentos e (4) a alocação dos conteúdos nos agrupamentos. Dada a preocupação do algoritmo em definir a melhor descrição para o agrupamento e como o objetivo do trabalho é a promoção de uma melhor visualização do domínio de gestão de ideias, o Lingo apresenta-se como uma boa alternativa. Após o processo de agrupamento, todos os dados ficam armazenados na ontologia formando a base de conhecimento e permitindo posteriormente a

\footnotetext{
${ }^{1}$ http://project.carrot2.org/
} 
avaliação por um especialista de domínio sobre a mesma, ou seja, o resultado obtido com a aplicação do algoritmo retroalimenta a ontologia de domínio. $\mathrm{Na}$ etapa de explicitação do conhecimento é feita a análise dos dados, buscando identificar padrões e tendências que auxiliem no momento da escolha das ideias para implementação. Os dados armazenados na ontologia servem de suporte à geração de redes/mapas que auxiliam no entendimento do domínio, compondo a base de conhecimento. De maneira geral, as redes interconectam os termos mais importantes, que representam os rótulos dos agrupamentos, com as ideias pertencentes ao agrupamento.

\section{APRESENTAÇÃO DOS RESULTADOS}

A construção do cenário de aplicação envolveu a coleta de 55 (cinquenta e cinco) ideias, de forma aleatória, presentes no site da empresa Starbucks $\AA^{2}$ com o objetivo de evidenciar relações e tendências de mercado da empresa. A escolha da base se deu por motivos de estruturação do formato de disposição das ideias, bem como a mesma ter sido utilizada em outros cenários de estudos, envolvendo o domínio de gestão de ideias como nos estudos de Westerski, Dalamagas e Iglesias (2013). O período de coleta e extração dos dados para compor a base de dados ocorreu no final do ano de 2014.

A categoria escolhida para aplicação do algoritmo foi o domínio de novas tecnologias. Para todas as ideias coletadas construiu-se um documento XML correspondente, elaborado manualmente, objetivando a semiestruturação textual. A estrutura do documento XML pode ser observada na Figura 2.

A Figura 4 apresenta a nuvem dos principais termos presentes na base de ideias. Pode-se observar que o nome da empresa é o mais citado, seguido pelos termos app, windows phone, drinks e cofffe. Pela frequência dos termos observa-se que existe um forte apelo por parte dos usuários por aplicativos mobile para Windows Phone ${ }^{\circledR}$ voltado a drinks e coffee.

${ }^{2}$ http://mystarbucksidea.force.com/apex/idealist?lsi=0 


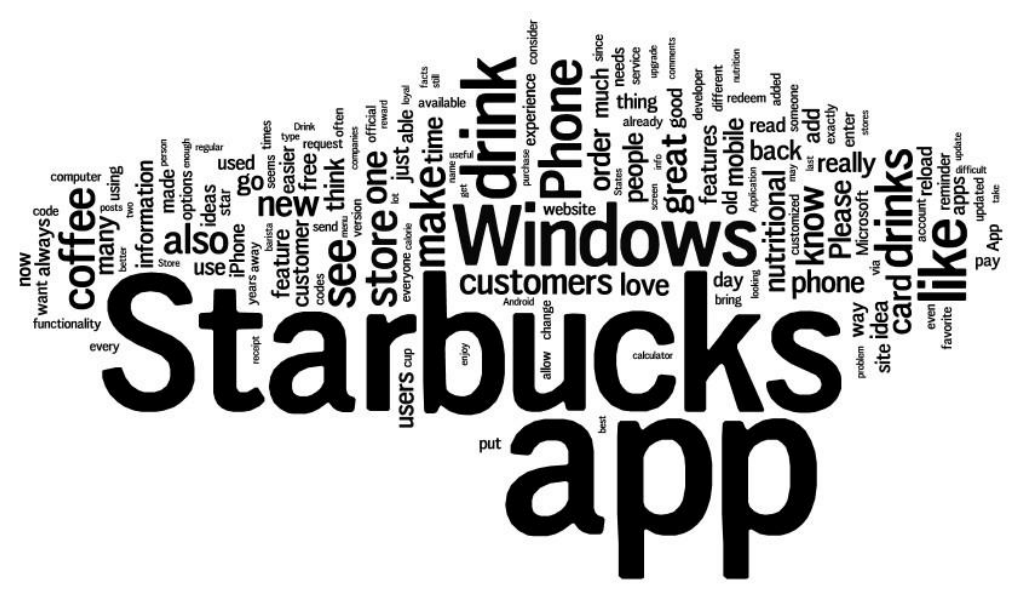

Figura 4 - Nuvem dos termos mais significativos do conjunto de ideias

Fonte: Autores

A análise somente pelos termos mais significativos do conjunto não possui maior expressividade e nem robustez, pois analisa apenas a frequência com que os termos são citados, contribuindo pouco no entendimento do domínio.

A partir da análise de agrupamento é possível explorar o conteúdo de grandes volumes de dados permitindo que o especialista de domínio analise grupos de objetos e não somente um único objeto. A análise de agrupamento auxilia também na detecção de objetos incomuns e os resultados podem ser utilizados para tomada de decisão empresarial.

O modelo apresentado no trabalho promove suporte a identificação das principais e mais coocorrentes ideias presentes no domínio de análise, facilitando assim a visualização da base de dados. A pesquisa visa demonstrar a efetividade do modelo por meio da formação dos agrupamentos.

A Figura 5 apresenta os agrupamentos obtidos a partir da aplicação do protótipo desenvolvido. Pode-se observar que o agrupamento com maior representatividade é o de cor verde, denominado de Starbucks App for Windows Phone, seguido pelos agrupamentos Other Topics (cor roxa), Favorite Drink (cor azul), Mobile App (cor amerela) e New App (cor azul). As palavras em maiúsculas correspondem a descrição do agrupamento formado a partir do algoritmo lingo, e as palavras mínusculas ao título da ideia. 


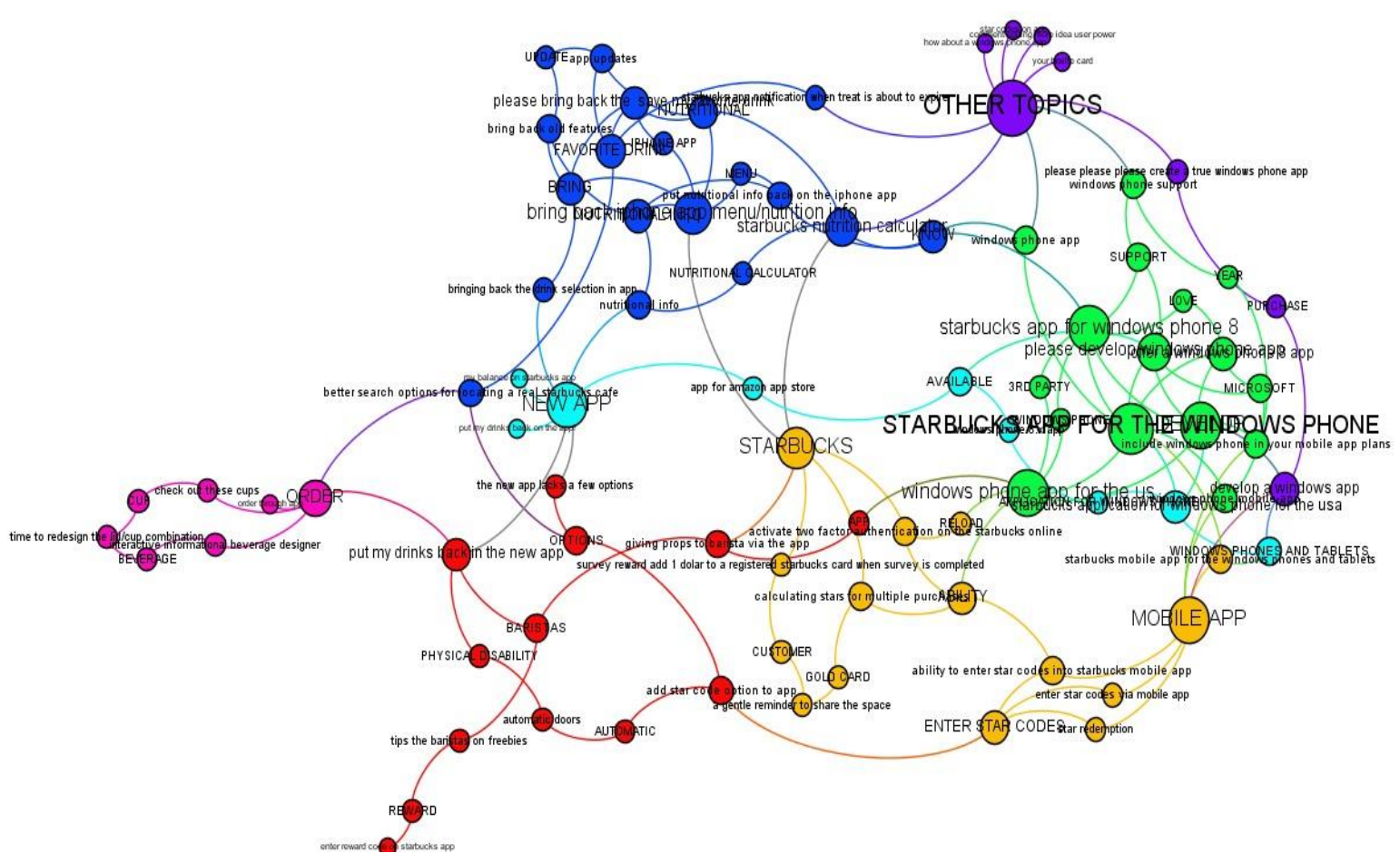

Figura 5 - Agrupamentos formados a partir do domínio de análise Fonte: Autores

Ao analisar as informações expressas na formação dos agrupamentos, ocorre uma melhora no entendimento do domínio de análise de ideias. Pela expressividade das descrições dos agrupamentos, a visualização e interpretação são facilitadas.

Pode-se observar que existe um apelo por parte da comunidade de colaboradores da empresa que fornecem ideias, por novos aplicativos mobile para windows phone principalmente na versão 8 , evidência esta comprovada pelos agrupamentos em cor roxa, azul escuro, verde, amarelo e azul claro. Observa-se ainda que os colaboradores desejam aplicativos voltados para 0 termo drinks. A questão nutricional é um fator de destaque presente no agrupamento azul escuro através do interesse em aplicativos que forneçam informações nutricionais ou ainda calculadoras nutricionais.

Via a aplicação do protótipo desenvolvido observou-se que os cinco termos mais representativos no domínio em análise, presentes na

Tabela 1, são: 
Tabela 1 - Termos mais coocorrentes do domínio de análise

\begin{tabular}{cc}
\hline Termo & $\begin{array}{c}\text { Número de vezes em que aparecem } \\
\text { nos documentos (ideias) }\end{array}$ \\
\hline APP & 38 \\
Phone & 20 \\
Drink & 18 \\
Store & 15 \\
Windows & 15 \\
\hline
\end{tabular}

Fonte: Autores

A partir dos termos mais significativos optou-se por selecionar os dois principais termos, no caso, "App" e "Windows Phone". Apesar dos termos phone e windows aparecem separados na tabela, os dois representam o segundo par de termos que mais coocorre no domínio.

Na Figura 6 é apresentado o agrupamento obtido a partir de uma consulta à base de conhecimento para projetar a imagem em função da query de pesquisa "App".

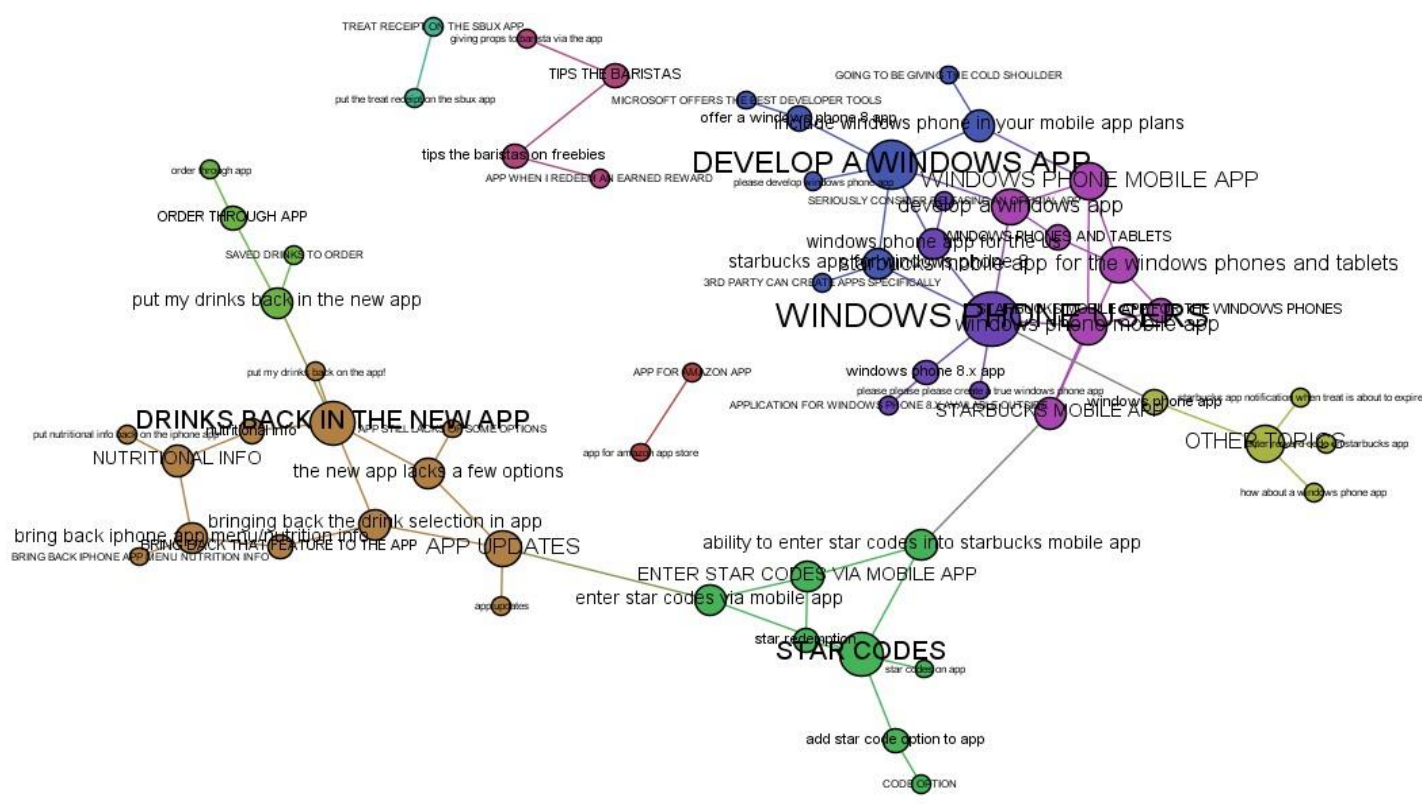

Figura 6 - Agrupamento formado a partir da query App

Fonte: Autores 
Neste cenário foram identificados quarenta documentos contendo a query "APP", sendo agrupados em dez agrupamentos. Pode-se observar ainda que relacionado ao domínio de "App" os colaboradores da organização solicitam mais aplicativos relacionados ao domínio de "Drinks back in the new $A p p^{\prime \prime}$, representado pelo agrupamento na cor marrom, o desenvolvimento de aplicativos para "windows", apresentado pelo agrupamento na cor azul escuro e aplicativos para usuário de "windows phone", representado pelo agrupamento na cor roxo sendo este o agrupamento mais representativo no domínio de análise.

Ainda no domínio de "Drinks back in the new App", observa-se que existe um forte apelo por aplicativos relacionados com a questão nutricional. Informação esta que confirma o que foi apresentado na Figura 5, contendo o agrupamento do conjunto de análise como um todo. As descrições do agrupamento são relacionadas a informações nutricionais para aplicativos para Iphone e a questão do cálculo nutricional.

Na Figura 7 é apresentado o agrupamento obtido com a aplicação do algoritmo Lingo com a query "Windows Phone".

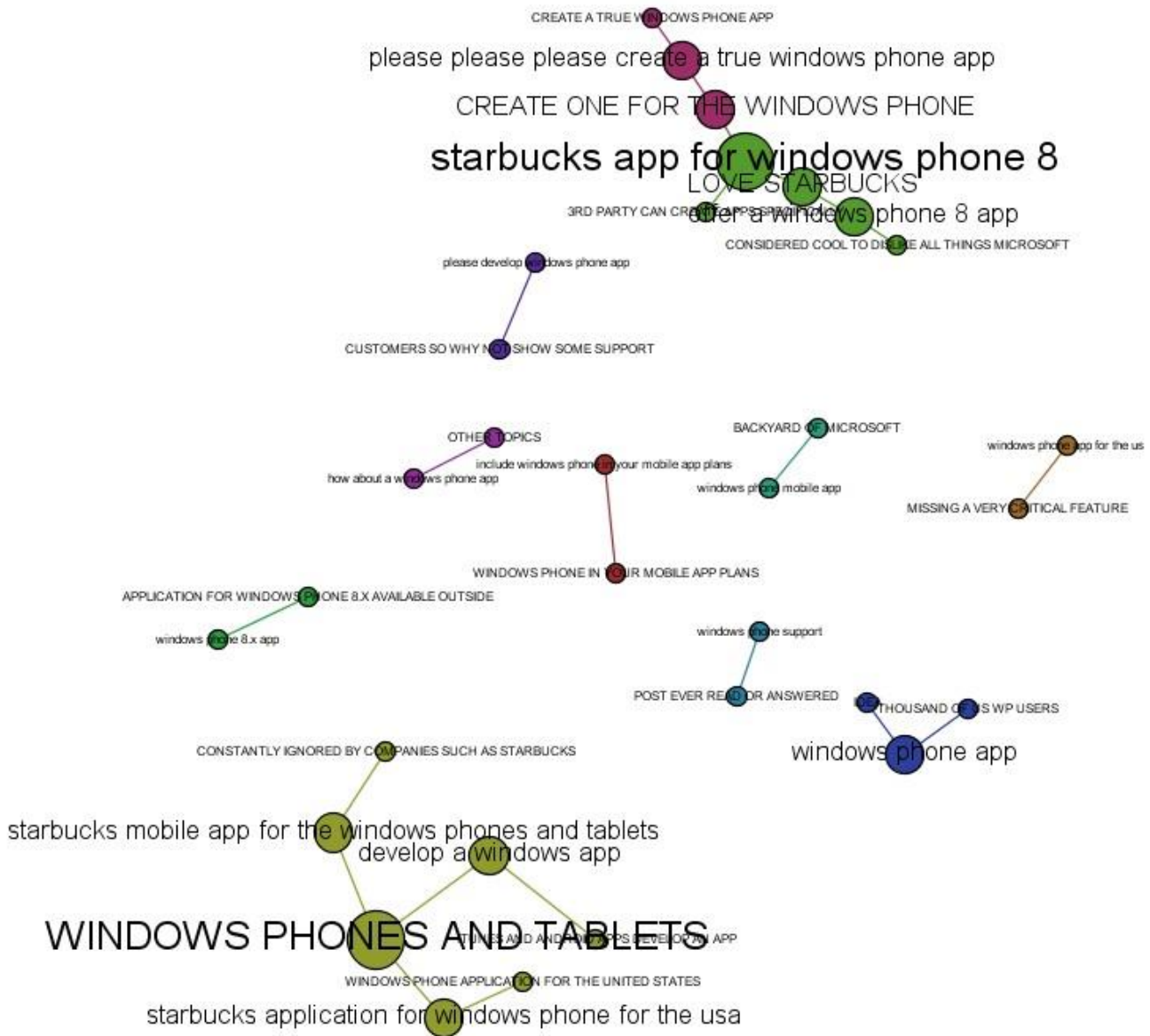




\section{Figura 7 - Cenário envolvendo a classificação da query "Windows Phone"}

Fonte: Autores

Neste segundo cenário foram identificados quinze documentos contendo a query "Windows Phone", sendo agrupados em onze agrupamentos. Pode-se observar a relação deste domínio com o domínio de aplicativos móveis, representado na Figura 7 pelo agrupamento na cor azul escuro com o termo "Windows Phone App".

Neste caso observa-se que o agrupamento mais expressivo é o em cor verde, cuja descrição solicita o desenvolvimento de aplicativos para celulares

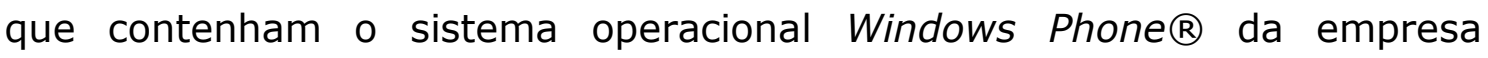
Microsoft $\AA$, e o desenvolvimento de aplicativos para tablets. Outra característica da formação deste agrupamento é que os colaboradores da

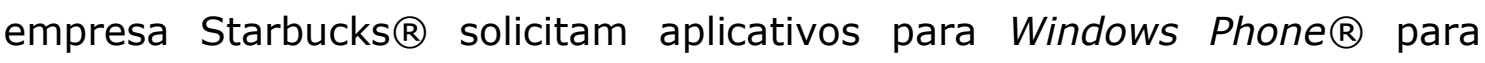
usuários dos EUA.

Após a apresentação dos cenários e análise dos mesmos, pode-se observar que por meio da aplicação da análise de agrupamento, padrões e tendências podem ser evidenciados e melhor visualizados por especialistas com 0 objetivo de auxiliar na tomada de decisão.

\section{CONSIDERAÇÕES FINAIS}

Neste trabalho foi proposto um modelo baseado em ontologia e na análise de agrupamento para auxiliar no processo de tomada de decisão no domínio de gestão de ideias. Para tanto se desenvolveu uma ontologia de domínio para representação do conhecimento e para prover suporte à representação semântica das ideias. A representação semântica auxilia no processo de agrupamento de ideias em categorias, na busca e na avaliação das ideias. Com isto é possível extrair as ideias mais fortemente discutidas.

Por meio da semântica é possível definir as regras para combinar ideias e seus relacionamentos, promovendo a representação dos relacionamentos. O uso de ontologias possibilita o raciocínio, a análise e a atuação sobre o conhecimento armazenado. 
Adicionalmente, o modelo considera técnicas de análise multivalorada. Essas técnicas permitem avaliar um conjunto de características em relação a uma população em análise. Uma das técnicas de análise multivalorada mais utilizada é a análise de agrupamento. A análise de agrupamento é uma técnica que possibilita agrupar objetos com base nas semelhanças de cada observação. A ideia central na formação dos agrupamentos é manter a homogeneidade dentro do grupo formado e a heterogeneidade entre os grupos. Com a aplicação da técnica de agrupamento é possível reduzir as informações de um conjunto de " $n$ " indivíduos para informações de um novo conjunto de " $n$ " grupos, onde os " $n$ " grupos são significativamente menores em relação aos " $n$ " indivíduos.

Dada esta premissa pode-se observar que por meio da análise de agrupamento é possível evidenciar padrões e tendências com relação às ideias presentes no domínio em análise. Uma ideia analisada isoladamente pode não representar um potencial de implementação, porém quando analisada em conjunto com outras ideias, pode evidenciar uma possível tendência ou demanda de mercado. Agrupamentos permitem que usuários interpretem de uma melhor maneira as ideias coletadas na organização. Com isto o processo de tomada de decisão é facilitado, auxiliando especialistas a decidirem sobre quais ideias são passíveis de serem implementadas.

As informações presentes na base de conhecimento permitem a formação e apresentação de dos grupos de ideias possibilitando que especialistas aloquem melhor o tempo dispendido na análise de tendências e demandas apontadas por clientes e colaboradores

Com a aplicação do modelo proposto ao cenário de pesquisa envolvendo a empresa Starbucks ${ }$, observou-se que existe um forte apelo para o desenvolvimento de aplicativos mobile que utilizam o sistema operacional Windows Phone ${ }^{\circledR}$, por aplicativos que forneçam informações nutricionais ou ainda calculadoras nutricionais, e por aplicativos que relacionados ao domínio de bebidas.

Como trabalhos futuros pretende-se aplicar outros algoritmos de agrupamento com o objetivo de analisar qual a melhor abordagem para lidar com informações textuais. Além disso, o aprimoramento das análises dos mapas produzidos pelo processo de agrupamento através da análise de redes sociais é algo desejável. 


\section{REFERÊNCIA BIBLIOGRÁFICA}

Andrienko, G., Andrienko, N. (2009) Interactive cluster analysis of diverse types of spatiotemporal data. Acm Sigkdd Explorations Newsletter, New York, 11 (2), 19-28.

Artz, K. W., et al. (2010) A longitudinal study of the impact of R\&D, patents, and product innovation on firm performance. Journal of Product Innovation Management, 27 (5), 725-740.

Badawy, M. K. (2011) Technovation - Is open innovation a field of study or a communication barrier to theory development: A perspective. Technovation, $31(1), 65-67$.

Barbieri, J. C., Álvares, A. C. T., \& Cajazeira, J. E. R. (2009) Gestão de ideias para inovação contínua. Porto Alegre: Bookman.

Baregheh, A., Rowley, J., \& Sambrook S. (2009) Towards a multidisciplinary definition of innovation. Management Decision, United Kingdom, 47 (8), 1323-1339.

Bautzer, D. (2009) Inovação: repensando as organizações. São Paulo: Atlas.

Bessant, J., Lamming, R., Noke, H., \& Phillips, W. (2005) Managing innovation beyond the steady state. Technovation, Amsterdam, 25 (12), 1366-1376.

Björk, J., \& Magnusson, M. G. (2009) Where do good innovation ideas come from? Exploring the influence of network connectivity on innovation idea quality. Journal of Product Innovation Management, Malden, 26 (6), 662-670.

Björk, J., Boccardelli, P., \& Magnusson, M. G. (2010) Ideation capabilities for continuous innovation. Creativity \& Innovation Management, Malden, 19 (4), 385-396.

Blohm, I., Bretschneider, U.; Leimeister, J. M., \& Krcmar, H. (2011) Does collaboration among participants lead to better ideas in IT-based idea competitions? An empirical investigation. International Journal of Networking and Virtual Organisations, Olney, 9 (2), 106-122.

Boeddrich, H. J. (2004) Ideas in the workplace: a new approach towards organizing the fuzzy front end of the innovation process. Creativity and Innovation Management, Malden, 13 (4), 274-285.

Borst, W. N. (1997) Construction of engineering ontologies for knowledge sharing and reuse. Universiteit Twente. 
Bothos, E., Apostolou, D., \& Mentzas, G. (2012) Collective intelligence with webbased information aggregation markets: The role of market facilitation in idea management. Expert Systems with Applications, Amsterdam, 39 (1), 13331345.

Brem, A., \& Voigt, K. I. (2007) Innovation management in emerging technology ventures: the concept of an integrated idea management. International Journal of Technology, Policy and Management, Olney, 7 (3), 304-321.

Carpineto, Claudio et al. (2009) A survey of web clustering engines. ACM Computing Surveys, New York, 41 (3).

Chesbrough, H. (2006) Open Innovation: the new imperative for creating and profiting from technology. Harvard Business School.

Cicyt, Comisión Interministerial De Ciencia y Tecnología. (2007) Plan Nacional de Investigación Científica, Desarrollo e Innovación Tecnológica 2008-2011, $1(1)$.

Conry-Murray. (2010). Can Enterprise Social Networking Pay Off? Internet Evolution. [Online]. Disponível em: http://www.internetevolution.com/document.asp?doc_id=173854\&page_nu mber=2; Acesso em: Jan, 2015.

Cruz, C. D., Carneiro, P. C. S. (2006) Modelos biométricos aplicados ao melhoramento genético. 2.ed. Viçosa: UFV, 585.

Endesley, S. (2010) Innovation in action: a pratical guide for healthcare teams. London: BMJ Books.

Fernandes, R. P.; Grosse, I. R.; Krishnamurty, S.; Witherell, P.; Wileden, J. C. (2011) Semantic methods supporting engineering design innovation. Advanced Engineering Informatics, 25 (2), 185-192.

Gambatese, J. A.; Hallowell, M. (2011) Enabling and measuring innovation in the construction industry. Construction Management and Economics, 29 (6).

Gibson, R.; Skarzynsky, P. (2008) Inovação: prioridade no 1: o caminho para transformação nas organizações. Tradução: Alessandra Mussi Araujo, Rio de Janeiro: Elsevier.

Gómez-Pérez, A. (1999) Ontological engineering: A state of the art.Expert Update: Knowledge Based Systems and Applied Artificial Intelligence, 2 (3), 33-43.

Govindarajan, V., \& Trimble C. (2012) Innovation: create far from home, win everywhere. Boston: Harvard Business Review.

Gruber, T. R. (1995) Toward principles for the design of ontologies used for knowledge sharing? International journal of human-computer studies, 43 (5), 907-928. 
Guarino, N. (1998) Formal Ontology in Information Systems: Proceedings of the 1st International Conference June 6-8, 1998, Trento, Italy.

Hüsig, S., \& Kohn, Stefan. (2011) "Open CAI 2.0"-Computer Aided Innovation in the era of open innovation and Web 2.0. Computers in Industry, 62 (4), 407-413.

Jain, A. K.; Murty, M. N.; Flynn, P. J. (1999) Data clustering: a review. Acm Computing Surveys, 31 (3), 264-323.

Kobashi, N. Y. (2007) Fundamentos semânticos e pragmáticos da construção de instrumentos de representação de informação. DataGramaZero - Revista de Ciência da Informação, 8 (6).

Lula, P., \& Paliwoda-Pękosz, G. (2008) An Ontology-Based Cluster Analysis Framework. Proceedings Of The First International Workshop On Ontologysupported Business Intelligence, New York, 1-6.

Magnusson, P. R., Netz J., \& Wästlund E. (2014) Exploring holistic intuitive idea screening in the light of formal criteria, Technovation, 34 (5-6), 315-326.

Neches, R., Fikes, R., Finin, T., Gruber, T., Patil, R., Senator, T., \& Swartout, W. (1991) Enabling Technology for Knowledge Sharing. In AI Magazine, Fall.

OECD. (2010) The OECD Innovation Strategy: getting a head start on tomorrow. Paris, France.

Osinski, S. \& Weiss, D. (2004) Conceptual clustering using Lingo algorithm: evaluation on open directory project data. In: INTERNATIONAL IIS: IIPWM' 04 CONFERENCE, 2004, Zakopane, Poland. Proceedings...Zakopane, Poland, 369-378.

Poveda, G., Westerski, A., \& Iglesias, C. A. (2012) Application of semantic search in Idea Management Systems. International Conference for Internet Technology And Secured Transactions, 230 - 236.

Reid, S. E., \& Brentani, U. (2004) The fuzzy front end of new product development for discontinuous innovation: a theoretical model. Journal of Product Innovation Management, Malden, 21 (3), 170-184.

Silva, D. L., Demarques, E. A., Souza, R. R., \& Lima, G. A. B. O. (2009) Ontologias e Unified Modeling Language: uma abordagem para representação de domínios de conhecimento. Datagramazero - Revista de Ciência da Informação, 10 (5).

Sint, R.; Markus, M.; Schaert, S.; Kurz, T. (2010) Ideator - a collaborative enterprise idea management tool powered by KiWi. Fifth Workshop "Semantic Wikis. Linking Data and People". Hersonissos, Greece.

Studer, R., Benjamins, V. R., \& Fensel, D. (1998) Knowledge engineering: principles and methods. Data \& knowledge engineering, 25 (1), 161-197. 
Tidd, J., Bessant, J., \& Pavitt, K. (2005) Gestão da Inovação. 3a. Ed. Porto Alegre: Bookman, 600.

Trott, P. (2012) Gestão da inovação e desenvolvimento de novos produtos. 4. ed. Porto Alegre: Bookman.

Vandenbosch, B., Saatcioglu, A., \& Fay, S. (2006) Idea management: a systemic view. Journal of Management Studies, Malden, 43 (2), 259-288.

Vasconcelos, E. S.; Cruz, C. D., Bhering, L. L.; Resende Júnior, M. F. R. (2007) Método alternativo para análise de agrupamento. Pesq. agropec. bras., Brasília , 42 (10).

Vygotsky, L. S. (2007) A formação social da mente. 7 ed. São Paulo: Martins Fontes.

Westerski, A.; Iglesias, C. A. (2011) Exploiting Structured Linked Data in Enterprise Knowledge Management Systems: An Idea Management Case Study. EDOCW. p. 395-403, IEEE Computer Society.

Westerski, A., Dalamagas, T., \& Iglesias, C. A. (2013) Classifying and comparing community innovation in Idea Management Systems, Decision Support Systems.

Westerski, A., Iglesias, C. A., \& Rico, F. T. (2010) A Model for Integration and Interlinking of Idea Management Systems. 4th Metadata and Semantics Research Conference (MTSR 2010), Alcalá de Henares, Spain.

Witell, L., Löfgren, M., \& Gustafsson, A. (2011) Identifying ideas of attractive quality in the innovation process, The TQM Journal, 23 (1), 87-99.

Xie, L., \& Zhang, P. (2010) Idea Management System for Team Creation. Journal of Software, North America, 5 nov. 